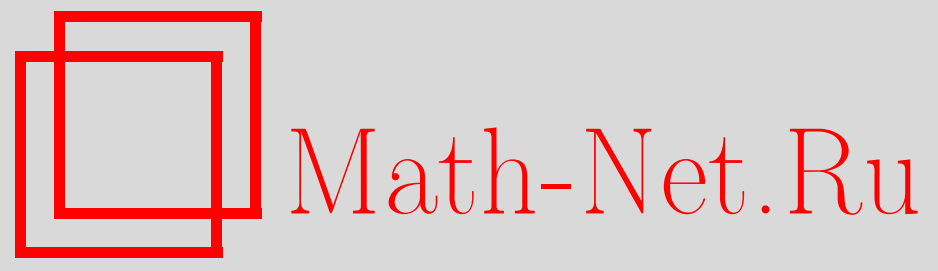

В. А. Колмыков, Инертные матрицы и паросочетания в частично ориентированных деревьях, Дискрет. матем., 2003, том 15, выпуск 4, 119-125

DOI: https://doi.org/10.4213/dm220

Использование Общероссийского математического портала Math-Net.Ru подразумевает, что вы прочитали и согласны с пользовательским соглашением http://www . mathnet.ru/rus/agreement

Параметры загрузки:

IP : 34.239 .49 .27

26 апреля 2023 г., 18:16:59

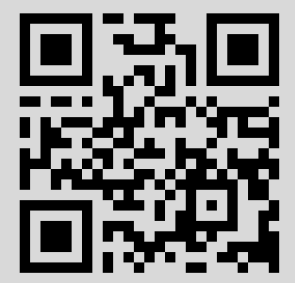


УдК 519.17

\title{
Инертные матрицы и паросочетания в частично ориентированных деревьях
}

\author{
() 2003 г. В. А. Колмыков
}

\begin{abstract}
Исследуется понятие инертности матрицы, то есть независимости ее вырожденности или невырожденности при заменах ненулевых элементов на произвольные ненулевые числа. В частично ориентированных графах рассматриваются неориентированные паросочетания. Обсуждается одна квантовомеханическая модель, при изучении которой удобно использовать такие паросочетания. Доказано, что совершенные и несовершенные частично ориентированные деревья (то есть имеющие и, соответственно, не имеющие совершенного паросочетания) могут быть построены из элементарных при помощи некоторых операций, то есть множество совершенных деревьев и множество несовершенных деревьев являются свободными конечнопорожденными алгебраическими структурами.
\end{abstract}

\section{1. Молекулы углеводородов и графы}

Квантовомеханическая модель молекулы углеводорода чрезвычайно сложна. Ее исследование представляет собой значительную часть современной квантовой химии.

Многие фрагменты этой модели (даже в довольно простых ситуациях) не поддаются исследованию точными математическими методами и в настоящее время изучаются в различных приближениях. Так обстоит дело, например, с расчетом энергии $\pi$-электронов. В результате некоторых приближений и замен переменных, задача о спектре оператора Гамильтона сводится (подробнее об этом см., например, главу 12 в [1] или главу 8 в [2]) к определению спектра некоторой $(0,1)$-матрицы $A$. Эта матрица является матрищей смежности графа (о свойствах матриц смежности см., например, [2]), который строится следуюшим образом. Его вершины - это атомы углерода, две различные вершины соединены ребром, если в молекуле имеется связь между соответствующими атомами. Погрешности приближений, приводящих к такой модели, вероятно, невелики, коль скоро свойства модели довольно хорошо согласуются с экспериментом.

В беседах с химиками об адекватности такой модели, однако, всегда присутствует (иногда и значительный) скептицизм по поводу этих погрешностей (см. также часть 2 тома 1 в [3]). Более естественным представляется рассмотрение некоторой матрицы $\tilde{A}$, устроенной следующим образом. На диагонали стоят нули; если связи $i$-го и $j$-го атомов нет, то $\tilde{a}_{i j}=0$; если же связь есть, то числа $\tilde{a}_{i j}$ и $\tilde{a}_{j i}$ (для нас сейчас не важно, каков их квантовомеханический смысл) некоторым образом характеризуют эту связь.

Математик также не должен довольствоваться надеждой на возможную и еще не доказанную незначительность погрешностей, тем более, что в некоторых ситуациях свойства 
модели не зависят от величины этих погрешностей. Невырожденность матрицы $A$ интерпретируется как устойчивость молекулы (см. §8.1 в [2]). Оказывается, если в молекуле нет циклов, то вырожденность-невырожденность матришы $\tilde{A}$ не зависит от замены ненулевых элементов на любые ненулевые.

\section{2. Инертные матрицы}

Последнее утверждение предыдущего пункта следует сформулировать в виде точной теоремы. Для этого введем несколько новых понятий.

Вырожденную (соответственно, невырожденную) матрицу назовем инертно вырожденной (соответственно, инертно невырожденной), если при любой замене ее ненулевых элементов на любые ненулевые числа получается вырожденная (соответственно, невырожденная) матрица. Если матрица инертно вырождена или инертно невырождена, то назовем ее инертной.

Пример 1. Пусть $a, b, c$ и $d$ - некоторые ненулевые комплексные числа. Матрища $\left(\begin{array}{ll}a & b \\ 0 & 0\end{array}\right)$ инертно вырождена, $\left(\begin{array}{ll}a & b \\ c & 0\end{array}\right)$ инертно невырождена, $\left(\begin{array}{ll}a & b \\ c & d\end{array}\right)$ не инертна.

Пусть $M$ - комплексная $\boldsymbol{n} \times \boldsymbol{n}$ матриша. Поставим ей в соответствие $\boldsymbol{n}$-вершинный граф $G_{M}$ (заметим, что в статъе под графом мы понимаем пару $(V, E)$, где $V$ - конечное множество вершин, а множество ребер $E$ - некоторое симметрическое подмножество в $V^{2}$ ) следующим образом. Множество его вершин есть $\{1, \ldots, n\}$; вершины $i$ и $j$ не соединены ребром, если и только если $m_{i j}=m_{j i}=0$. Например, если $m_{i i} \neq 0$, то в графе присутствует петля в вершине $i$.

Матрицу $M$ назовем древесной, если $G_{M}$ - дерево.

Теорема 1. Всякая древесная матрича инертна.

Это утверждение непосредственно следует из теоремы 3.

Теорема 2. Матрича М инертно вырождена тогда и только тогда, когда все слагаемые определителя $|M|$ нулевые.

Матрича М инертно невырождена тогда и только тогда, когда ровно одно слагаемое определителя $|M|$ ненулевое.

Доказательство. В матрице $M$ всякий ненулевой элемент $m_{i j}$ заменим комплексной переменной $x_{i j}$. Полученную матрицу обозначим $X_{M}$. Определитель $\left|X_{M}\right|$ - это некоторый многочлен. Он имеет степень, не большую единицы, по каждой переменной $x_{i j}$.

Все слагаемые определителя $|M|$ равны нулю тогда и только тогда, когда многочлен $\left|X_{M}\right|$ нулевой. Ровно одно слагаемое определителя $|M|$ не равно нулю тогда и только тогда, когда многочлен $\left|X_{M}\right|$ является одночленом.

Матрица $M$ инертно вырождена тогда и только тогда, когда многочлен $\left|X_{M}\right|$ принимает нулевые значения для любых ненулевых значений переменных. Последнее равносильно тому, что многочлен $\left|X_{M}\right|$ принимает нулевые значения для любых значений переменных. Это значит, что он нулевой.

Матрища $M$ инертно невырождена тогда и только тогда, когда многочлен $\left|X_{M}\right|$ принимает лишь ненулевые значения при любых ненулевых значениях переменных (такой многочлен назовем категорическим).

Пусть комплексный многочлен $f$ имеет степень, не большую единищы, по каждой переменной. Докажем, что $f$ является категорическим тогда и только тогда, когда он 
является одночленом. Пусть $f$ имеет степень 1 по переменной $x$, множество остальных переменных обозначим $Y$. Тогда $f(x, Y)=x g(Y)+r(Y)$, где $g$ - ненулевой многочлен.

Покажем, что $r$ - нулевой многочлен. Действительно, если $r$ - ненулевой многочлен, то и $g r$ ненулевой. Пусть $Y_{0}$ - набор ненулевых значений переменных из $Y$ такой, что $g\left(Y_{0}\right) r\left(Y_{0}\right) \neq 0$. Тогда $f\left(-r\left(Y_{0}\right) / g\left(Y_{0}\right), Y_{0}\right)=0$, что противоречит категоричности $f$.

Итак, $f(x, Y)=x g(Y)$. Поэтому $g$ - категорический многочлен. Продолжая процесс вынесения переменной и далее, получим, что $f=c x y \ldots z-$ одночлен.

Ненулевые слагаемые определителя $|M|$ имеют ясную геометрическую интерпретацию. Матрице $M$ поставим в соответствие орграф $\vec{G}_{M}$ с множеством вершин $\{1, \ldots, n\}$ и множеством дуг $\left\{\overrightarrow{i j} \mid m_{i j} \neq 0\right\}$ (в частности, если $m_{i i} \neq 0$, то в $i$-й вершине имеется дуга $\overrightarrow{i i}$ ). Напомним, что дугой $\overrightarrow{u v}$ называется упорядоченная пара (не обязательно различных) вершин $u$ и $v$. Контуром длины $k \geqslant 2$ в орграфе называется чередующаяся последовательность $\left\{v_{1}, \overrightarrow{v_{1} v_{2}}, v_{2}, \ldots, \overrightarrow{v_{k-1} v_{k}}, v_{k}, \overrightarrow{v_{k} v_{1}}\right\}$ вершин и дуг орграфа, в которой $v_{i} \neq v_{j}$, если $i \neq j$. Контуром длины 1 в орграфе называется последовательность $\{v, \vec{v}\}$. Множество $F$ контуров называется совершенным 2-фактором, если всякая вершина орграфа принадлежит ровно одному контуру из этого множества.

Следующая лемма (или очень близкая к ней) независимо открывалась многими авторами. Мы приводим ее доказательство, так как другие источники или труднодоступны, или используют иную терминологию.

Лемма 1. Существует взаимно-однозначное соответствие между совершенными 2-факторами орграфа $\vec{G}_{M}$ и ненулевыми слагаемыми определителя $\mid$ М|.

Доказательство. Формально говоря, слагаемое определителя - это пара $\left(\sigma, m_{\sigma}\right)$, где $\sigma \in S_{n}, m_{\sigma}=(-1)^{i n v(\sigma)} m_{1, \sigma(1)} \ldots m_{n, \sigma(n)}$. Говорят, что это слагаемое соответствует подстановке $\sigma$. При такой формализации все $n$ ! слагаемых определителя различны между собой.

Слагаемое определителя считается равным нулю, если вторая его составляющая равна нулю. Совершенному 2-фактору $F$ естественно соответствует подстановка $\sigma_{F}$ множества вершин рассматриваемого орграфа, а именно, $\sigma_{F}(i)=j$ тогда и только тогда, когда $\overrightarrow{i j}$ принадлежит одному из контуров, составляющих $F$. Слагаемое определителя $|M|$, соответствующее этой подстановке, ненулевое, так как $m_{i, \sigma_{F}(i)} \neq 0$ для любого $i$.

Построенное соответствие между совершенными 2-факторами и ненулевыми слагаемыми определителя и является искомым. Обратное соответствие строится так. Пусть $\left(\sigma, m_{\sigma}\right)$ - ненулевое слагаемое определителя $|M|$. Разложим подстановку $\sigma$ в каноническое произведение непересекающихся циклов. Каждому такому циклу соответствует контур в $\vec{G}_{M}$ (в этот контур входят дуги вида $\left.\overrightarrow{i \sigma(i)}\right)$.

Теорема 3. Матрича М инертно вырождена тогда и только тогда, когда в орграфе $\vec{G}_{M}$ нет совершенного 2-фактора. Матрича $M$ инертно невырождена тогда и только тогда, когда в орграфе $\vec{G}_{M}$ есть ровно один совершенный 2-фактор. 


\section{3. Частично ориентированные графы}

Граф называется частично ориентированным, если некоторые его ребра ориентированы. В частности, всякий граф является частично ориентированным (ориентированы ребра из пустого множества ребер). Отметим, что петлю мы рассматриваем как частный случай ребра, но если на ребре с различными конщами существует две возможности ориентации, то для петли такая возможность одна.

Пусть $M$ - комплексная $n \times n$ матрица. Поставим ей в соответствие частично ориентированный $n$-вершинный граф $\vec{G}_{M}$ следующим образом. Множество его вершин естъ $\{1, \ldots, n\}$. Вершины $i$ и $j$ соединены неориентированным ребром, если $m_{i j} m_{j i} \neq 0$, дугой $\overrightarrow{i j}$, если $m_{i j} \neq 0$ и $m_{j i}=0$, не соединены, если $m_{i j}=m_{j i}=0$.

Паросочетанием в частично ориентированном графе назовем множество неориентированных ребер такое, что всякая вершина графа является концом не более, чем одного ребра из этого множества. Таким образом, здесь мы имеем дело с некоторым обобщением классического (см. [4]) понятия паросочетания. Частично ориентированный граф назовем совершенным, если в нем сушествует совершенное паросочетание, то есть такое, что всякая вершина графа является конщом ровно одного ребра из этого паросочетания. Заметим, что совершенные, несовершенные, полусовершенные неориентированные графы довольно хорошо изучены (см. [4]).

Теорема 4. Пусть $\vec{G}_{M}$ - частично ориентированное дерево. Матрица $M$ инертно вырождена тогда и только тогда, когда $\vec{G}_{M}$ несовериенно. Матрица М инертно невырождена тогда и только тогда, когда $\vec{G}_{M}$ совершенно.

Доказательство. Если $\vec{G}_{M}$ - частично ориентированное дерево, то существует взаимнооднозначное соответствие между его совершенными паросочетаниями и совершенными 2-факторами орграфа $\vec{G}_{M}$. Если в $\vec{G}_{M}$ существует совершенное паросочетание, то оно единственно, поскольку оно соответствует совершенному паросочетанию в дереве $G_{M}$. Теперь утверждение теоремы 4 следует из теоремы 3.

\section{4. Внутреннее строение}

Теперь опишем внутреннее строение совершенных и несовершенных частично ориентированных деревьев. Первая часть теоремы 5 очевидна, и мы приводим ее лишь для полноты формулировки. Неожиданной является вторая часть, которая утверждает, что все несовершенные частично ориентированные деревья и только они могут быть получены при помощи трех элементарных деревьев и трех операций (это несколько напоминает то, как векторы конечномерного пространства получаются из базисных векторов и двух операций).

Ниже используются четыре элементарных дерева - это 1-, 2-, 3- и 4-вершинные деревья, соответственно, $K_{1,0}, K_{1,1}, K_{1,2}$ и $K_{1,3}$ (здесь $K_{m, n}$ - стандартное обозначение для полного двудольного $(n+m)$-вершинного графа), а также четыре многозначные (точнее, конечнозначные) операции:

бинарная коммутативная операция -, состоящая в соединении двух частично ориентированных деревьев мостом (например, $K_{1,0}-K_{1,2}$ является двухэлементным множеством, состояшим из $K_{1,3}$ и цепи $\left.P_{4}\right)$; 
бинарная коммутативная операция $\rightarrow$, состоящая в соединении двух частично ориентированных деревьев ориентированным мостом (например, $K_{1,0} \rightarrow K_{1,2}$ является четырехэлементным множеством, которое состоит из двух частично ориентированных цепей, получающихся из $P_{4}$ произвольным ориентированием одного концевого ребра, и двух частично ориентированных деревьев, получающихся из $K_{1,3}$ произвольным ориентированием одного ребра);

бинарная коммутативная операция ·, состоящая в склейке двух частично ориентированных деревьев по вершине (например, $K_{1,1} \cdot K_{1,2}$ является двухэлементным множеством, состоящим из $K_{1,3}$ и цепи $P_{4}$ );

унарная операция $\uparrow$, состоящая в произвольном ориентировании (всех, нескольких или ни одного) неориентированных ребер частично ориентированного дерева (например, $\uparrow\left(K_{1,2}\right)$ является шестиэлементным множеством).

Говорят, что объект $T$ получается из объектов $T_{1}, \ldots, T_{k}$ при помощи операций $O_{1}, \ldots, O_{s}$, если $T$ принадлежит множеству, описываемому некоторой (правильно составленной с учетом арности) формулой, в которой участвуют только объекты $T_{1}, \ldots, T_{k}$ (возможно, многократно) и только операции $O_{1}, \ldots, O_{s}$ (также, возможно, многократно).

Теорема 5. Все совершенные частично ориентированные деревья и только они получаются из $K_{1,1}$ при помощи операчий $-u \rightarrow$.

Все несовершенные частично ориентированные деревья и только они получаются из $K_{1,0}, K_{1,2}$ и $K_{1,3}$ при помощи операчий $\cdot \rightarrow u \uparrow$.

Доказательство. То, что всякое совершенное частично ориентированное дерево может быть получено указанным способом, следует непосредственно из определения совершенности. То, что указанным способом получаются только совершенные частично ориентированные деревья, следует из замкнутости множества совершенных частично ориентированных деревьев относительно операций - и $\rightarrow$.

Множество всех частично ориентированных деревьев, которые можно получить указанным во втором утверждении теоремы способом, обозначим через $\mathscr{L}$. Так как $K_{1,0}, K_{1,2}$ и $K_{1,3}$ несовершенны и множество несовершенных частично ориентированных деревьев замкнуто относительно операций $\cdot, \rightarrow$ и $\uparrow$, все элементы $\mathscr{X}$ несовершенны.

Оставшуюся часть доказательства разобьем на фрагменты. Порядком частично ориентированного дерева здесь называется число его вершин.

(1) Перебор показывает, что несовершенные частично ориентированные деревья порядка, не превосходящего 4, принадлежат $\mathscr{X}$.

(2) Частично ориентированное дерево нечетного порядка несовершенно.

(3) Вершина степени 1 называется концевой. Предконцевой вершиной назовем вершину, инцидентную хотя бы одной концевой и ровно одной не концевой. Дерево не имеет предконцевых вершин тогда и только тогда, когда это дерево есть $K_{1, n}$.

(4) Если $n \neq 1$, то $\uparrow\left(K_{1, n}\right) \subset \mathscr{X}$.

(5) Индукцией по порядку $m$ частично ориентированного несовершенного дерева $T$ докажем, что оно принадлежит $\mathscr{L}$. 
Если $m \leqslant 4$, то см. (1).

Пусть $m \geqslant 5$ и $T \in \uparrow\left(K_{1, n}\right)$. Тогда см. (4).

Пусть $m \geqslant 5$ и $T \notin \uparrow\left(K_{1, n}\right)$. В таком случае $T$ имеет предконщевую вершину. Удалим из него эту вершину и все конщевые вершины, инцидентные ей. Полученное частично ориентированное дерево обозначим $T_{1}$. Если же из $T$ удалить только концевые вершины, инцидентные рассматриваемой предконцевой, то оставшееся частично ориентированное дерево обозначим $T_{2}$ (его порядок на 1 больше, чем порядок $T_{1}$ ). Ясно, что $T \in T_{1} \cdot Y$ и $T \in T_{2} \cdot Z$, где $Y \in \uparrow\left(K_{1, l}\right)$ и $Z \in \uparrow\left(K_{1, l-1}\right)$ (здесь $l$ - степень рассматриваемой предконцевой вершины).

Если $l \geqslant 3$, то, согласно (4), $Y, Z \in \mathscr{X}$. Либо $T_{1}$, либо $T_{2}$ нечетного порядка, и согласно (2) несовершенно. Все участвующие в операциях частично ориентированные деревья имеют порядок меньший, чем порядок $T$. Отсюда, учитывая предположение индукщии, заключаем, что $T \in \mathscr{X}$.

Рассмотрим случай $l=2$. Если $T_{1}$ несовершенно, то, учитывая предположение индукции, из включения $T \in T_{1} \cdot Y$ и (4) заключаем, что $T \in \mathscr{X}$. Если же $T_{1}$ совершенно, то $T_{2}$ несовершенно (см. (2)). Ребро, соединяюшее концевую и предконцевую вершины, является ориентированным (иначе $T$ было бы совершенным). Поэтому $T \in T_{2} \rightarrow K_{1,0}$. Отсюда, учитывая предположение индукции, заключаем, что $T \in \mathscr{X}$.

\section{5. Перспективы и открытые вопросы}

1. Матричная модель (или, что почти то же самое, графовая модель) молекулы углеводорода является фундаментальной концепцией. Поэтому она естественно появляется при различных способах упрощения квантовомеханической модели. Автор является сторонником исследования матричной модели на более ранних стадиях, чем это обычно принято, и предлагает для этого использовать понятия инертных матриц и частично ориентированных графов.

2. Имеются ситуации, в которых приближения, приводящие к матрице смежности, недопустимы. Например, равенство $a_{i j}=a_{j i}$ обусловлено симметрией $i$-го и $j$-го атомов углерода и соответствующей симметрией электронных облаков, но при наличии сильных воздействий симметрия может быть нарушена. Другой пример, расчеты, основанные на квантовомеханической модели, являются вероятностными: система большую часть времени находится в состоянии, описываемом некоторой матрицей $B$, которая является результатом известных усреднений матриц $B(P, t)$ (где $P$ - некоторый набор параметров, a $t$ - время); поэтому, если нас интересуют свойства матриц $B(P, t)$, то мы не можем применять приближения, фактически сводящие $B(P, t)$ к постоянной матрище. При исследовании подобных маргинальных состояний могут быть полезны свойства матриц, сильно отличающихся от матрищы смежности (например, несимметричных).

3. В спектральной теории графов естественным и даже необходимым (см., например, [5]) является исследование спектральных свойств матриц более сложных, чем матрица смежности. При таких рассмотрениях естественно появляются ориентированные ребра. В этом смысле ориентированное ребро является лишь математической абстракцией, не требующей химического истолкования. Существует ли такое истолкование - это открытый вопрос, ответ на который могли бы найти специалисты по соответствующим разделам математического моделирования. 
4. Легкость доказательства теоремы 4 обусловлена единственностью (в случае существования) совершенного паросочетания в частично ориентированном дереве и соответствия таких паросочетаний совершенным 2-факторам некоторого орграфа. Было бы интересно обобшить теорему 5, то есть описать (в терминах некоторых элементарных графов и некоторых операций) строение множества всех орграфов, не имеющих совершенных 2-факторов и имеющих ровно один совершенный 2-фактор.

\section{Список литературы}

1. Фудзинага С., Метод молекуляриых орбиталей. Мир, Москва, 1983.

2. Цветкович Д., Дуб М., Захс Х., Спектры графов. Теория и применение. Наукова Думка, Киев, 1984.

3. Синаноглу О., Современиая квантовая химия. тт. 1, 2. Мир, Москва, 1968.

4. Ловас Л., Пламмер М., Прикладные задачи теории графов. Теория паросочетаний в математике, физике, химии. Мир, Москва, 1998.

5. Stankevich I. V., Skvortsova M. I., Kolmykov V. A., Subbotin V. F., Mnuchin V. B., Spectral graph theory in chemistry. Mathematical methods in contemporary chemistry. Gordon \& Breach, New York, 1996, 101-141.

Статья поступила 13.12.2001, Переработанный вариант поступил 08.10.2002. 